\title{
A Case of Miller Fisher Syndrome with Unusual Features: Brisk Muscle Stretch Reflexes and Facial Palsy
}

Yuzhu Tang ${ }^{1 *}$, Khadija A. Mamsa ${ }^{1}$, Asok K. Lahiri ${ }^{2}$, Zev Carrey ${ }^{1}$, Richard L. Petrillo ${ }^{1}$ and Dariush Alaie ${ }^{1}$

${ }^{1}$ Department of Medicine, Mount Vernon Hospital, Mount Vernon, NY 10550, USA

${ }^{2}$ Departement of Neurology, Mount Vernon Hospital, Mount Vernon, NY 10550, USA

\begin{abstract}
Miller-Fisher syndrome (MFS), a rare variant of Guillain-Barré syndrome (GBS), typically presents as the triad of areflexia, ophthalmoplegia, and ataxia. We report a case of MFS associated with brisk muscle stretch reflexes and facial palsy which may confuse the diagnosis of MFS.
\end{abstract}

Keywords: Miller-Fisher Syndrome; Anti-GQ1b; Ophthalmoplegia; Ataxia; Muscle stretch reflexes; Facial palsy

\section{Introduction}

Miller-Fisher syndrome (MFS), a variant of Guillain-Barré syndrome (GBS), first described by Dr. Miller Fisher consists of the triad of ophthalmoplegia, ataxia, and areflexia [1]. In its classical form, the condition is easily diagnosed. Here we report a case of MFS which showed in addition to classical features association of facial palsy and exaggerated reflexes, both of which are very rare in this condition.

\section{Case Presentation}

A 64 year old male came to emergency room complaining of headache, double vision and "wobbly" gait for one day. He had single vision if he only used one of his eyes. Patient had past medical history of hypertension, COPD and arthritis. He had mild diarrhea for 3 days and bronchitis about 10 days prior to admission for which he was still on doxycycline. Patient had mild cough and nasal congestion on presentation to the emergency room.

On physical examination, his blood pressure was 143/103 mm Hg, heart rate was $66 / \mathrm{min}$, and respiratory rate was $20 / \mathrm{min}$. Patient was alert, and oriented to time, place and person. Pupils were equal and reactive to light. On cranial nerve examination, there was paralysis of left eye abduction, suggestive of left sixth cranial nerve palsy. Muscle stretch reflexes were $2+$ in all four extremities. Plantar response was flexor, and Romberg test was negative. There was no focal motor weakness, sensory deficit or in coordination of upper limbs. The heel-knee shin test was mild impaired, but gait unsteadiness. Patient managed to walk a few steps with ataxic gait. The unsteady gait was initially thought to be due to diplopia, so his left eye was patched, however, there was no improvement of ataxia.

Head CT showed a normal finding except mild left sphenoid sinusitis. Electrocardiogram showed sinus rhythm, heart rate 73 , no heart block. WBC was $11.2 \times 10^{9} / \mathrm{L}$ and erythrocyte sedimentation rate was $18 \mathrm{~mm} / \mathrm{h}$.

Next day, patient complained of worsening of all symptoms. His vision was no longer double, rather blurred. On examination both eyes were fixed in forward gaze positions with no ocular movement. Pupils were equal, but reaction to light was sluggish compared to previous day. Muscle stretch reflexes were brisk $3+$ bilateral extremities and remained so until discharge. Right facial muscle weakness was noted after two days and right upper eyelid was sensitive to touch. Patient was unable to walk at all due to his worsening ataxic gait in the following few days.

Ophthalmology evaluation did not reveal optical and retinal changes, but confirmed the total bilateral external ophthalmoplegia and sluggish pupillary light reflex.

Magnetic resonance imaging of head demonstrated several areas of increased signal in the white matter in the centrum semiovale and periventricular areas, compatible with microvascular ischemia. There was no mass effect or evidence of an acute infarct. Mucoperiosteal thickening was noted in the right sphenoid sinus, compatible with chronic sinusitis, Carotid doppler had no sign of carotid stenosis. Tensilon test did not produce obvious changes in eye movement, which made myasthenia gravis unlikely. Rapid plasma reagin for syphilis was negative. Serum glucose was $86 \mathrm{mg} / \mathrm{dl}$, and hemoglobin A1c was $6.2 \%$. The titer of Serum antineutrophil cytoplasmic (ANCA) IgG was within normal reference rang. Lumbar puncture and CSF analysis showed: protein $39.44 \mathrm{mg} / \mathrm{dl}$, glucose $64 \mathrm{mg} / \mathrm{dl}$, Cells: 0/ul, culture was negative. Serum Lyme titer was within reference range. Elevated serum antiganglioside GQ1b titer 43\% supported the clinical diagnosis of Miller Fisher syndrome. The reference range of anti-GQ1b is $<20 \%$ in the tested laboratory.

The patient was treated with IVIG for 5 days at 3 days after the onset of the symptoms. On day 3 of IVIG, patient had minimal improvement in headache and facial muscle weakness. After IVIG completion, patient had some improvement of ataxic gait. He could manage to walk a few steps with unsteady gait. The blurred vision showed no improvement 6 days following IVIG or 9 days after onset. Patient was discharged to short-term rehabilitation and followed by his primary care physician and neurologist. At discharge, he was able to walk with moderate

*Corresponding author: Yuzhu Tang, Department of Medicine, Mount Vernon Hospital, $12 N 7^{\text {th }}$ Ave, Mount Vernon 10550, USA, Tel: (914) 631-6441; Fax: (914)371-1184; E-mail: yuzhut@hotmail.com

Received December 19, 2011; Accepted June 21, 2012; Published June 23 , 2012

Citation: Tang Y, Mamsa KA, Lahiri AK, Carrey Z, Petrillo RL, et al. (2012) A Case of Miller Fisher Syndrome with Unusual Features: Brisk Muscle Stretch Reflexes and Facial Palsy. J Neurol Neurophysiol 3:135. doi:10.4172/2155-9562.1000135

Copyright: ( 2012 Tang Y, et al. This is an open-access article distributed under the terms of the Creative Commons Attribution License, which permits unrestricted use, distribution, and reproduction in any medium, provided the original author and source are credited. 
ataxia. He had total external ophthalmoplegia, mild facial weakness. There was no limb weakness. Muscle stretch reflexes were still brisk.

The blurred vision gradually resolved by two months after the onset of symptoms. There was noticeable vertical eye movement, but no horizontal movement. The gait was minimally broad based without any ataxia. At the three-month follow-up by his neurologist, the patient had no gait impairment, and eye movement improved except for left lateral rectus weakness and the patient experienced double vision on left lateral gaze. The rest of neurological examination was normal. The stretch reflexes were $2+$. Subsequent information from patient's primary care doctor at $6^{\text {th }}$ month revealed that the patient has recovered completely without any abnormalities in eye movement or gait. During the entire course of the hospitalization and clinic follow-ups, there was no disturbance of consciousness. Electrophysiologic evaluation, cervical spine MRI and laboratory study on serum vitamin B12 and copper level were not performed.

\section{Discussion}

We present this case because of the association of MFS with facial palsy and brisk reflexes which may confuse the diagnosis. MFS is a relatively uncommon condition; therefore it is mandatory to rule out other conditions with rapid onset of ophthalmoplegia and ataxia, such as brainstem stroke, Wernick's encephalopathy, Bickerstaff brainstem encephalitis and also other acute painful ophthalmoplegias such as bilateral cavernous sinus thrombosis, Tolosa-Hunt syndrome and superior orbital fissure syndrome. Painful ophthalmoplegias were not considered because they present with clearly distinctive features and MRI abnormalities. Brainstem stroke with total ophthalmoplegia was easily excluded because most patients will be in coma with abnormal MRI. Our patient had no history of alcohol abuse or nutritional deficiency, so Wernick's encephalopathy was not considered. Bickerstaff brainstem encephalitis was considered because of the clinical presentation multiple cranial nerve palsy and brisk reflexes, but it also seemed unlikely due to absence of any disturbance of consciousness or limb weakness, and normal MRI and CSF study $[2,3]$. Coincidence of cervical myelopathy causing brisk reflexes was ruled out clinically because of no leg weakness, no Babinski sign and no progression as well as normalization of muscle stretch reflexes on recovery though MRI of cervical spine was not performed. Multiple diabetic cranial nerve palsy was not seriously considered because total bilateral ophthalmoplegia is extremely unlikely, and our laboratory work-ups ruled out diabetes.

So in our case the presentation of sudden onset ataxia, ophthalmoplegia with elevated Anti-GQ1b most likely diagnosis remains MFS despite the two uncommon associations of brisk reflexes and facial palsy.

Over $90 \%$ of MFS patient have positive anti-GQ1b IgG antibodies which is correlated with ophthalmoplegia, the most prevalent symptom in MFS [4-6]. The positive anti-GQ1b antibody helps in diagnosis of MFS, but it is not specific to MFS [2]. The classical triads of ophthalmoplegia, ataxia and areflexia relative common in MFS, whereas facial palsy is only seen in approximately $22 \%$ of patients. Brisk reflexes are seldom seen in MFS. Recent studies of Japanese GBS patients found that normal or exaggerated muscle stretch reflexes can be seen in $10-13 \%$ GBS cases which are associated with anti-GM1 antibody and milder form of disease $[7,8]$. The presence of brisk reflexes in its variant, MFS, even though rare, should not be used to exclude the diagnosis of MFS.

The treatment for GBS, including MFS, is supportive care combined with plasmapheresis or intravenous immune globulin (IVIG). IVIG has proved to be as effective as plasma exchange in GBS treatment [912]. Many believe that plasmapheresis or IVIG treatment can shorten the recovery period by $40-50 \%[10,12]$. However, a study of 92 cases of MFS suggests that IVIG or plasmapheresis only has minimal efficacy in hastening the recovery of MFS. It seems not to change the final outcome of MFS [13].

The natural course of untreated Guillain-Barré syndrome is progressive in the first two weeks, followed by a plateau of 2-4 weeks. In most cases the recovery is underway after 3-4 weeks of onset. The time for full recovery varies significantly from a few weeks to a few years [11]. The median range of MFS recovery without immunotherapy is 4 (range 1 to 185) months. The usual order of recovery of findings is ataxia gait, followed by ophthalmoplegia, and areflexia [5]. In our patient, the improvement of the ataxic gait started at about one week upon finishing IVIG treatment. There was remarkable improvement of ophthalmologia by three months with complete recovery by six month; there was no hypo-/ or areflexia during the entire course of clinic follow-up.

\section{References}

1. Fisher M (1956) An unusual variant of Acute Idiopathic Polyneuritis (Syndrome of Ophthalmoplegia, Ataxia and Areflexia). N Engl J Med 255: 57-65.

2. Ito M, Kuwabara S, Odaka M, Misawa S, Koga M, et al. (2008) Bickerstaff's brainstem encephalitis and Fisher syndrome form a continuous spectrum: clinical analysis of 581 cases. J Neurol 255: 674-682.

3. Odaka M, Yuki N, Yamada M, Koga M, Takemi T, et al. (2003) Bickerstaff's brainstem encephalitis: clinical features of 62 cases and a subgroup associated with Guillain-Barré syndrome. Brain 126: 2279-2290.

4. Carpo M, Pedotti R, Lolli F, Pitrola A, Allaria S, et al. (1998) Clinical correlate and fine specificity of anti-GQ1b antibodies in peripheral neuropathy. J Neurol Sci 155: 186-191.

5. Mori M, Kuwabara S, Fukutake T, Yuki N, Hattori T (2001) Clinical features and prognosis of Miller Fisher syndrome. Neurology 56: 1104-1106.

6. Chiba A, Kusunoki S, Obata H, Machinami R, Kanazawa I (1993) Serum anti-GQ1b IgG antibody is associated with ophthalmoplegia in Miller Fisher syndrome and Guillain-Barré syndrome: clinical and immunohistochemical studies. Neurology 43: 1911-1917.

7. Kuwabara S, Ogawara K, Koga M, Mori M, Hattori T, et al. (1999) Hyperreflexia in Guillain-Barré syndrome: relation with acute motor axonal neuropathy and anti-GM1 antibody. J Neurol Neurosurg Psychiatry 67: 180-184.

8. Yuki N, Kokubun N, Kuwabara S, Sekiguchi Y, Ito M, et al. (2011) GuillainBarré syndrome associated with normal or exaggerated tendon reflexes. J Neurol 259: 1181-1190.

9. Winer JB (2002) Treatment of Guillain-Barré syndrome. QJM 95: 717-721.

10. (1985) Plasmapheresis and acute Guillain-Barré syndrome. The Guillain-Barré syndrome Study Group. Neurology 35: 1096-1104.

11. Ropper AH, Wijdicks EFM, Truax BT (1991) Guillain-Barré syndrome Philadelphia. FA Davis.

12. Hughes RA, Wijdicks EF, Barohn R, Benson E, Cornblath DR, et al. (2003) Practice parameter: immunotherapy for Guillain-Barré syndrome: report of the Quality Standards Subcommittee of the American Academy of Neurology. Neurology 61: 736-740.

13. Mori M, Kuwabara S, Fukutake T, Hattori T (2007) Intravenous immunoglobulin therapy for Miller Fisher syndrome. Neurology 68: 1144-1146. 